\title{
Association of cord blood methylation fractions at imprinted insulin-like growth factor 2 (IGF2), plasma IGF2, and birth weight
}

\author{
Cathrine Hoyo $\cdot$ Kimberly Fortner $\cdot$ Amy P. Murtha $\cdot$ \\ Joellen M. Schildkraut • Adelheid Soubry • Wendy Demark-Wahnefried • \\ Randy L. Jirtle · Joanne Kurtzberg • Michele R. Forman • \\ Francine Overcash $\cdot$ Zhiqing Huang $\cdot$ Susan K. Murphy
}

Received: 4 August 2011/Accepted: 16 February 2012/Published online: 6 March 2012

(C) The Author(s) 2012. This article is published with open access at Springerlink.com

\begin{abstract}
Purpose Altered methylation at Insulin-like Growth Factor 2 (IGF2) regulatory regions has previously been associated with obesity, and several malignancies including colon, esophageal, and prostate adenocarcinomas, presumably via changes in expression and/or loss of imprinting, but the functional significance of these DNA methylation marks have not been demonstrated in humans. We examined associations among DNA methylation at IGF2 differentially methylated regions (DMRs), circulating IGF2 protein concentrations in umbilical cord blood (UCB) and birth weight in newborns.

Methods Questionnaire data were obtained from 300 pregnant women recruited between 2005 and 2009. UCB
\end{abstract}

C. Hoyo · J. M. Schildkraut · F. Overcash

Department of Community and Family Medicine, Duke

University Medical Center, 2200 West Main Street, Ste. 600,

Durham, NC 27705, USA

C. Hoyo $\cdot$ J. M. Schildkraut $\cdot$ A. Soubry $\cdot$ S. K. Murphy

Duke Cancer Institute, Duke University Medical Center, 6036

Hock Plaza, 2424 Erwin Rd, Durham, NC 27710, USA

C. Hoyo $(\bowtie)$

Department of Obstetrics and Gynecology, Division of Clinical Epidemiology, School of Medicine, Duke University, 2200 West Main Street, Suite 600, Durham, NC 27705, USA

e-mail: cathrine.hoyo@duke.edu

K. Fortner · A. P. Murtha

Division Maternal Fetal Medicine, Department of Obstetrics and Gynecology, Duke University Medical Center, 4022 Hosp South, Durham, NC 27710, USA

W. Demark-Wahnefried

Department of Behavioral Sciences, UT M.D. Anderson Cancer Center, 1515 Holcombe Blvd., Unit 1340, Houston,

TX 77030-4009, USA
DNA methylation was measured by bisulfite pyrosequencing. UCB plasma concentrations of soluble IGF2 were measured by ELISA assays. Generalized linear regression models were used to examine the relationship between DMR methylation and IGF2 levels.

Results Lower IGF2 DMR methylation was associated with elevated plasma IGF2 protein concentrations $(\beta=$ $-9.87, p<0.01)$; an association that was stronger in infants born to obese women (pre-pregnancy BMI $>30 \mathrm{~kg} / \mathrm{m}^{2}$, $\beta=-20.21, p<0.0001)$. Elevated IGF2 concentrations were associated with higher birth weight $(p<0.0001)$ after adjusting for maternal race/ethnicity, pre-pregnancy BMI, cigarette smoking, gestational diabetes, and infant sex. These patterns of association were not apparent at the H19 DMR.

\section{R. L. Jirtle}

Department of Radiation Oncology, Duke University Medical Center, 139 Env Safety Bldg., Durham, NC 27710, USA

\section{J. Kurtzberg}

Pediatric Blood/Marrow Transplantation, Department of Pathology, Duke University Medical Center, 1430 N. Pavilion Bldg, Durham, NC 27705, USA

\section{R. Forman}

Department of Epidemiology, UT M.D. Anderson Cancer Center, 1515 Holcombe Blvd., Unit 1340, Houston,

TX 77030-4009, USA

\section{Z. Huang $\cdot$ S. K. Murphy}

Division Gynecologic Oncology, Department of Obstetrics and Gynecology, Duke University Medical Center, 226 B Wing LSRC 450 Research Drive, Durham, NC 27708, USA

Z. Huang $\cdot$ S. K. Murphy

Department of Pathology, Duke University Medical Center, Durham, NC 27708, USA 
Conclusion Our data suggest that variation in IGF2 DMR methylation is an important mechanism by which circulating IGF2 concentrations, a putative risk factor for obesity and cancers of the colon, esophagus, and prostate, are modulated; associations that may depend on pre-pregnancy obesity.

Keywords IGF2 * H19 · ELISA - DNA methylation · Epigenetics · Epidemiology

\section{Introduction}

The 'developmental origins of adult disease susceptibility' hypothesis posits that environmental exposures early in the development permanently alter the phenotype, influencing susceptibility to lifetime risk of obesity and several cancers [1]. These alterations are thought to be mechanistically driven by epigenetic alterations. The most stable and wellstudied epigenetic mechanism is DNA methylation, which involves the covalent attachment of a methyl group to the 5 -carbon position of the pyrimidine ring of cytosine residues when in $5^{\prime}-\mathrm{CpG}-3^{\prime}$ context. This epigenetic modification is faithfully transmitted to nascent DNA molecules during DNA replication and is maintained during somatic cell proliferation. DNA methylation contributes to the induction and maintenance of transcriptional gene silencing, propagating shifts in gene expression that orchestrate long-term susceptibility to several adenocarcinoma including colon [2, 3], ovary [4], prostate [5, 6], stomach [7], and esophagus [8]. Epigenetic mechanisms have also been hypothesized to link birth weight, a proxy for the wellbeing of the offspring in utero, with some of these cancers in adulthood [9-15]. However, genomic regions in which DNA methylation increases risk of these chronic diseases are still unknown. Ultimately, cancer prevention will require identification of aberrant epigenetic profiles driving risk, demonstration of their functional significance as well as environmental exposures associated with their perturbation and 'windows' of susceptibility during life's course.

Identification of aberrant DNA methylation marks has been hampered by a lack of stable targets with known baseline levels, allowing for deviations from baseline to be detected. The need for epidemiologic studies to use specimens obtained from non-invasive means, such as peripheral blood leukocytes or buccal cells, which are often the only tissues available in population-based studies, has presented additional hurdles; epigenetic regulation and dysregulation can vary by tissue and cell type. Genomically imprinted genes are among several major classes of genes that are regulated by DNA methylation [16]. Unlike most autosomal genes that follow Mendelian laws of inheritance where both parentally derived alleles are expressed, imprinted genes are monoallelically expressed, with their functional haploidy controlled by parental allele-specific DNA methylation at differentially methylated regions (DMRs). Because these methylation marks are stable covalent modifications that are established before germ layer specification [17], these methylation profiles should be maintained in all germ layers, resulting in quantifiable systemic homogeneity in locus-specific epigenetic regulation [17, 18]. As such, imprinted DMRs stably exhibit $\sim 50 \%$ methylation in somatic tissues.

Insulin-like-growth factor-2 (IGF2) is perhaps the most intensively studied imprinted gene and is paternally expressed, with at least two regulatory DMRs located upstream of exon 3 and upstream of neighboring maternally expressed $H 19$ [19]. Hypermethylation (methylation fractions higher than the expected $\sim 50 \%$ ) at the H19 DMR $[2,4]$ and $\mathrm{CpG}$ hypomethylation (methylation fractions lower than the expected $\sim 50 \%$ ) at the IGF2 DMR [2] have been associated with higher $I G F 2 \mathrm{mRNA}$ levels suggesting increased transcriptional activity, presumably via relaxation of imprint controls. Such increased transcriptional activity is a common feature in adenocarcinomas of the ovary, esophagus and prostate [4, 8, 20-25]. IGF2 DMR loss of methylation was observed in DNA that was obtained from peripheral blood leukocytes of adults exposed to severe caloric restriction periconeptionally, suggesting methylation shifts in response to adverse events occur early and are stable over many decades [26]. Based on these observations, methylation status at regulatory sequences of imprinted genes has been proposed as 'archives' or 'biosensors' of early exposure [27, 28]. However, the functional significance of these DMR methylation marks has not previously been demonstrated in epidemiologic studies. The objective of these analyses was to examine variation in DMR methylation at sequences regulating $I G F 2 / H 19$, in relation to UCB plasma IGF2 protein concentrations. We also determined whether IGF2 protein concentrations are associated with variation in birth weight, an indicator of maternal and fetal well-being.

\section{Methods}

\section{Study participants}

A detailed description of study participants has been previously reported [29]. Briefly, between April 2005 and June 2009, 1101 eligible pregnant women seen at one of two Durham, North Carolina, obstetric facilities were identified through appointment logs and were invited to enroll in the Newborn Epigenetics STudy (NEST), a multiethnic cohort study. Approximately $85 \%(n=940)$ provided written consent to participate. Eligibility criteria were as follows: 
age $\geq 18$ years, English speaking, and an intent to use one of two Durham County obstetric facilities which enabled specimen collection at delivery. We excluded women infected with HIV and those planning to have their newborns adopted. Women were identified through appointment logs of two prenatal clinics serving the above two obstetrics care facilities: Duke maternal fetal medicine (MFM) and Durham Regional Hospital Obstetrics, Durham County, NC, as part of the NEST. Gestational ages at enrollment were $19-42$ weeks (median $=28$ weeks). This report is limited to $300(32 \%)$ deliveries in whom DNA methylation analyses were performed and protein measurements are available. The 300 women are comparable to the 940 women with respect to maternal age (mean age 29 years), obesity (body mass index [BMI], $28 \mathrm{~kg} / \mathrm{m}^{2}$ ), and sex of offspring ( $49 \%$ men). This study was approved by the Institutional Review Board at Duke University Medical Center.

\section{Data collection}

\section{Questionnaire data collection}

A standardized questionnaire was either self-administered or completed with a trained interviewer. The questionnaire items included socio-demographic information including marital status, educational attainment, race/ethnicity, smoking history, and current smoking status, a history of common chronic diseases and conditions, dietary supplement use, weight and height before pregnancy (from which BMI and weight gain was computed). BMI was calculated as pre-pregnancy weight in kilograms divided by height in square meters $\left(\mathrm{kg} / \mathrm{m}^{2}\right)$, and weight gain was the difference between usual weight and weight at the delivery visit. At delivery, medical records were abstracted to verify cigarette smoking during pregnancy, reported morbidity and age, and to obtain additional parturition data including blood pressure and mode of delivery, body temperature and chorioamnionitis (the latter two as indicators of infection which may influence protein measurements), as well as offspring information including sex, anthropometric measurements, and 5-min Apgar scores.

\section{Specimen collection and processing}

Umbilical cord blood specimens were collected in vacutainer tubes containing EDTA within minutes of delivery and inverted gently to mix the anticoagulant with the blood. Specimens were stored in the hospital refrigerator until transported to the laboratory usually within $12 \mathrm{~h}$ of collection where they were centrifuged to isolate plasma and buffy coat, which was aliquotted and stored at $-80^{\circ} \mathrm{C}$. DNA was extracted from the white blood cells in the buffy coat using Puregene reagents according to the manufacturer's protocol (Qiagen, Valencia, CA).

\section{Methylation measurements}

Description of methylation measurements and primers used has been described elsewhere [30]. Briefly, cord blood samples from the first 438 subjects were analyzed for methylation status at two DMRs controlling the expression of $I G F 2$. The steps comprised modification of genomic DNA by treating it with sodium bisulfite using a highthroughput method previously described [4, 31] to convert unmethylated cytosines to uracils, leaving methylated cytosines unchanged. We then obtained a quantitative measure of methylation at each DMR using a Pyromark Q96 MD Pyrosequencing instrument (Qiagen; Valencia, CA). Three CpGs were measured at the IGF2 DMR [2], located upstream of the imprinted $I G F 2$ promoter in exon $3,[2,26]$ (chr 11p15.5; CpG site 1: 2,169,518; $\mathrm{CpG}$ site 2: 2,169,515; and $\mathrm{CpG}$ site 3: 2,169,499; NCBI Human Genome Build 37/hg19). We also measured DNA methylation at four $\mathrm{CpG}$ dinucleotides at the H19 DMR at a sequence motif that binds the CTCF zinc-finger protein (chr 11p15.5; CpG site 1: 2,024,261, CpG site 2: 2,024,259, $\mathrm{CpG}$ site 3: 2,024,257, and $\mathrm{CpG}$ site 4: 2,024,254; NCBI Human Genome Build 37/hg19). Methylation data were $98 \%$ complete. We validated both pyrosequencing assays using defined mixtures of plasmids containing the fully methylated or unmethylated versions of the bisulfate-modified sequence.

\section{Protein measurements}

Plasma concentrations of IGF2 were measured using microplate enzyme-linked immunosorbent assays (Diagnostic Systems Laboratories, Webster, TX). Procedures for measurement followed the manufacture's instructions. Absorbance was read using a microplate reader DSL, Webster, TX. The plasma IGF2 concentrations ranged from 50 to $2,000 \mathrm{ng} / \mathrm{ml}$, and assay sensitivity was $2.2 \mathrm{ng} / \mathrm{ml}$.

Statistical analyses

For analyses, maternal age at enrollment was categorized as 18-30, 30-39 versus 40 years or older; marital status was dichotomized into married/living with partner versus not married; educational level was categorized into groups defined as up to high school, some college to college graduate versus graduate/professional school; race/ethnicity was categorized into African Americans versus nonHispanic White versus other, cigarette smoking was dichotomized into smoking during pregnancy versus not smoking; birth weight was treated as both a continuous 
variable and dichotomized at less $<2500$ versus $\geq 2500 \mathrm{~g}$; maternal BMI was categorized into $<25,25-29,30-34$, and $35+\mathrm{kg} / \mathrm{m}^{2}$; weight gain during pregnancy was dichotomized at $<30$ pounds versus $\geq 30$ pounds; delivery mode was classified as vaginal vs. C-section; and histological evidence of chorioamnionitis was dichotomized as yes or no.

We examined the distribution of methylation fractions for normality at each $\mathrm{CpG}$ dinucleotide using KolmogorovSmirnov tests followed by principal components and confirmatory factor analyses to determine whether methylation at the three CpGs measured in the IGF2 DMR and the four CpGs measured at the H19 DMR were correlated to justify the use of a single mean for each DMR. Cronbach's alpha values for CpGs were 86 and $87 \%$ at the IGF2 DMR and H19 DMR, respectively. We also examined the distributions of birth weight, IGF2 proteins, maternal weight gain and usual BMI for normality and found that with the exception of maternal BMI and weight gain, these variables were normally distributed among the 300 participants under study. Thus, subsequent analyses dichotomized maternal BMI at $\leq 30 \mathrm{~kg} / \mathrm{m}^{2}$, and weight gain at the 75 th percentile.

The main goal of our analyses was to determine whether DNA methylation fractions at each DMR were associated with IGF2 protein concentrations. Although race/ethnicity and adult BMI have been previously associated with IGF2 concentrations and will be considered potential confounders, F-tests were used to identify additional potential confounders, by identifying factors associated with statistically significant variation $(p \leq 0.20)$ in IGF2 protein concentrations, and methylation levels at each DMR. In addition, chorioamnionitis was examined for potential effect modification and confounding as previous studies of predictors of IGF2 protein in otherwise healthy adults suggest acute infection may affect protein concentrations [32]. Mode of delivery was evaluated for potential confounding or effect modifiers also because animal data suggests DNA methylation patterns may vary by delivery mode [33]. Because $75 \%$ of African Americans in this study were $>30 \mathrm{~kg} / \mathrm{m}^{2}$ compared to $25 \%$ of Whites, we fitted linear regression models to examine the relationship between IGF2 protein and DMR methylation for African Americans and Whites and also for obese and non-obese women, separately. To further examine the association IGF2 DMR methylation and the protein product in the context of pre-pregnancy BMI or weight gain during the course of pregnancy, we repeated our analyses restricted to infants born to women $>30$ versus $\leq 30 \mathrm{~kg} / \mathrm{m}^{2}$ and also gain weight below and above the 75th percentile. Cross-product terms for maternal BMI and DNA methylation at each DMR were also computed and included in statistical models where differences were observed in stratified analyses. Only variables with $p$ value $<0.05$ were retained in final statistical models. To examine whether IGF2 protein concentrations were associated with birth weight, we also utilized linear regression models since birth weight was normally distributed. These latter models were adjusted for gestational diabetes, maternal BMI, race/ethnicity, and cigarette smoking, as these factors are known to independently influence birth weight in the general population. All statistical analyses were conducted in SAS version 9.3 (SAS Institute, Cary, $N C, U S A)$.

\section{Results}

IGF2 proteins and covariable

The average UCB plasma protein concentration of IGF2 among the newborns was $1029 \mathrm{ng} / \mu \mathrm{l}$, standard deviation $(\mathrm{SD}=458)($ Table 1$)$. Mean IGF2 concentration did not vary significantly by maternal age $(p=0.51)$, marital status $(p=0.82)$, smoking during pregnancy $(p=0.83)$, chorioamnionitis $(p=0.87)$ or indicators of maternal socioeconomic status such as educational attainment $(p=$ $0.32)$ and type of health insurance coverage $(p=0.22)$ although five individuals with no insurance had significantly lower IGF2 levels. We observed lower IGF2 concentration in infants who were male $(p$ value $=0.11)$, had low birth weight $(p$ value $=0.12$ ), those born by C-section ( $p$ value $=0.006)$ or to African American women ( $p$ value $=0.02)$. IGF2 concentrations were also lower in infants born to women with a pre-pregnancy BMI $>30$ $(p$ value $=0.06)$ and those for whom pregnancy weight gain was $<30 \mathrm{lbs}$ ( $p$ value $=0.09$ ).

\section{IGF2 and H19 DMR methylation and covariables}

Table 2 shows the distribution of DNA methylation levels by maternal and infant characteristics that were associated with variation in IGF2 protein concentrations in Table 1. Overall, average methylation levels among the newborns were $48.5 \%(\mathrm{SD}=7.7)$ and $61.4 \%(\mathrm{SD}=7.7)$ at the $I G F 2$ and $H 19$ DMRs, respectively. At the IGF2 DMR, methylation percentages were similar by race/ethnicity, sex, birth weight, delivery mode, weight gain during pregnancy, and BMI before pregnancy ( $p$ values $>0.20$ ) (Table 2). However, we observed slight methylation differences in infants born to women with and those without histological evidence of chorioamnionitis at delivery ( $p$ value $<0.18$ ); chorioamnionitis was considered for confounding in the $I G F 2$ and H19 DMR analyses as it exhibited significant methylation differences at $p \leq 0.20$, as were race/ethnicity, birth weight and three correlated factors; maternal BMI, weight gain during pregnancy and mode of delivery. 
Table 1 Distribution of offspring IGF2 concentrations by maternal and offspring characteristics

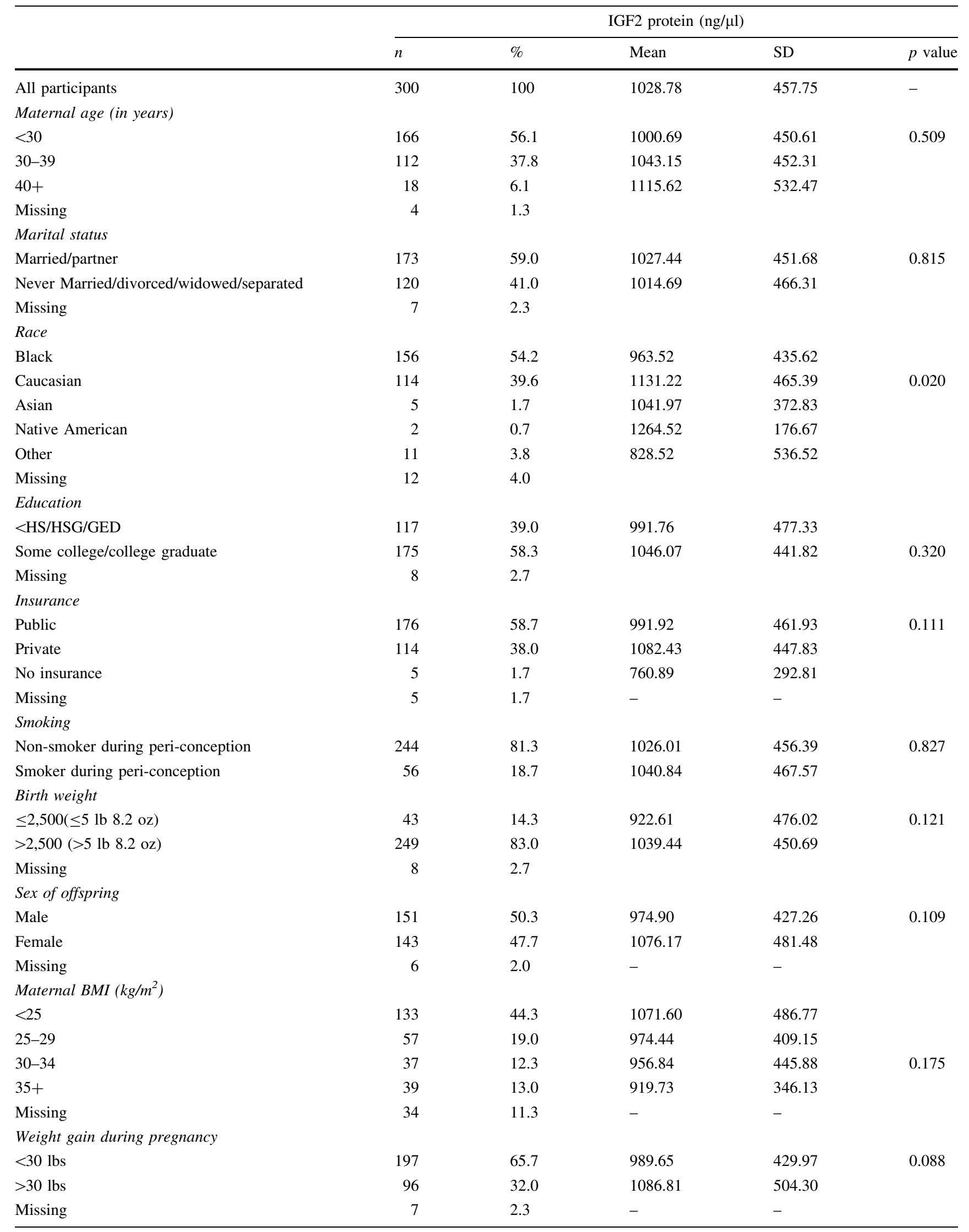


Table 1 continued

\begin{tabular}{|c|c|c|c|c|c|}
\hline & \multicolumn{5}{|c|}{ IGF2 protein $(\mathrm{ng} / \mu \mathrm{l})$} \\
\hline & $n$ & $\%$ & Mean & SD & $p$ value \\
\hline \multicolumn{6}{|c|}{ Delivery Route } \\
\hline Vaginal & 170 & 56.7 & 1086.13 & 451.76 & 0.006 \\
\hline C-section & 126 & 42.0 & 939.58 & 449.59 & \\
\hline Missing & 4 & 1.3 & - & - & \\
\hline \multicolumn{6}{|c|}{ Chorioamnionitis } \\
\hline Yes & 22 & 7.3 & 1044.34 & 397.05 & 0.869 \\
\hline No & 278 & 92.7 & 1027.55 & 462.82 & \\
\hline
\end{tabular}

Table $2 I G F 2$ and $H 19$ DMR methylation fractions and maternal and offspring characteristics

\begin{tabular}{|c|c|c|c|c|}
\hline & $I G F 2 \mathrm{DMR} \%(\mathrm{sd})$ & & $H 19$ DMR \% (sd) & $p$ value \\
\hline All participants & $48.5(7.7)$ & & $61.4(7.7)$ & - \\
\hline \multicolumn{5}{|l|}{ Racelethnicity } \\
\hline African American & $48.4(9.5)$ & & $62.1(8.3)$ & \\
\hline White & $48.2(5.3)$ & & $60.7(7.1)$ & \\
\hline Other & $50.0(5.6)$ & $p=0.742$ & $59.4(7.0)$ & $p=0.199$ \\
\hline \multicolumn{5}{|l|}{ Sex of offspring } \\
\hline Male & $48.4(6.7)$ & & $61.7(8.4)$ & \\
\hline Female & $48.3(8.8)$ & $p=0.289$ & $60.9(7.1)$ & $p=0.478$ \\
\hline \multicolumn{5}{|l|}{ Birth weight } \\
\hline$\leq 2,500(\leq 5 \mathrm{lb} 8.2 \mathrm{oz})$ & $48.6(9.4)$ & & $59.8(6.5)$ & \\
\hline$>2,500(>5 \mathrm{lb} 8.2 \mathrm{oz})$ & $48.3(7.5)$ & $p=0.875$ & $61.5(8.0)$ & $p=0.194$ \\
\hline \multicolumn{5}{|l|}{ Maternal BMI $\left(\mathrm{kg} / \mathrm{m}^{2}\right)$} \\
\hline$<25$ & $48.8(5.8)$ & & $60.7(6.6)$ & \\
\hline $25-29$ & $49.7(10.2)$ & & $61.2(7.4)$ & \\
\hline $30-34$ & $49.3(8.5)$ & & $63.0(9.6)$ & \\
\hline $35+$ & $47.5(6.2)$ & $p=0.618$ & $62.0(9.1)$ & $p=0.390$ \\
\hline \multicolumn{5}{|c|}{ Weight gain during pregnancy } \\
\hline$<30 \mathrm{lbs}$ & $48.7(8.7)$ & & $61.9(8.5)$ & \\
\hline$>30 \mathrm{lbs}$ & $47.9(4.8)$ & $p=0.487$ & $60.1(6.0)$ & $p=0.079$ \\
\hline \multicolumn{5}{|l|}{ Chorioamnionitis } \\
\hline Yes & $46.0(7.0)$ & & $58.8(5.9)$ & \\
\hline No & $48.7(7.7)$ & $p=0.180$ & $61.6(7.8)$ & $p=0.128$ \\
\hline \multicolumn{5}{|l|}{ Delivery route } \\
\hline Vaginal & 48.7 (8.9) & & $61.4(7.7)$ & \\
\hline C-section & $48.2(6.0)$ & $p=0.634$ & $61.3(7.9)$ & $p=0.950$ \\
\hline
\end{tabular}

H19 DMR methylation and IGF2 protein concentrations

Overall, we found a significant association between $H 19$ DMR methylation $(\beta=-8.08, p=0.02)$ and IGF2 protein concentrations (Table 3 ). However, adjusting for maternal BMI before pregnancy weakened this association ( $\beta=-4.69, p=0.07$ ) (data not shown), and additionally adjusting for chorioamnionitis and race/ethnicity further weakened this association $(\beta=-3.64, p=0.33)$. This association may be stronger in infants born to African Americans $(\beta=-6.90, p=0.15)$ than those to Whites $(\beta=-2.54, p=0.68)$, although neither reached statistical significance. However, BMI-specific analyses suggested this association may be more pronounced in infants born to non-obese women $(\beta=-8.52, p=0.09)$ compared to those born to obese women $(\beta=0.33, p=0.95)$. Further adjustment for BMI, weight gain, and mode of delivery did 
Table 3 Associations between methylation fractions at $I G F 2$ and H19 DMRs and IGF2 circulating concentrations stratified by race and maternal obesity

\footnotetext{
a Models determining whether methylation at the $H 19$ or $I G F 2$ DMRs predict protein levels. Models are adjusted in all participants, for birth weight, histological evidence of chorioamnionitis at delivery, maternal BMI before pregnancy and race. Models stratified by race/ethnicity and BMI were adjusted for birth weight, chorioamnionitis, and race/ ethnicity or maternal BMI before pregnancy
}

\begin{tabular}{|c|c|c|c|c|c|c|}
\hline & \multicolumn{3}{|c|}{ IGF2 $\mathrm{CpG}$ methylation } & \multicolumn{3}{|c|}{ H19 CpG methylation } \\
\hline & $\beta$ & SE & $p$ value & $\beta$ & SE & $p$ value \\
\hline \multicolumn{7}{|c|}{ All participants $(n=300)$} \\
\hline Crude & -9.87 & 3.90 & 0.01 & -8.08 & 3.51 & 0.02 \\
\hline Adjusted $^{\mathrm{a}}$ & -9.49 & 4.23 & 0.03 & -3.64 & 3.72 & 0.33 \\
\hline \multicolumn{7}{|c|}{ African Americans $(n=156)$} \\
\hline Crude & -7.94 & 4.23 & 0.06 & -10.37 & 4.35 & 0.02 \\
\hline Adjusted & -8.89 & 4.70 & 0.06 & -6.90 & 4.73 & 0.15 \\
\hline \multicolumn{7}{|c|}{ Whites $(n=114)$} \\
\hline Crude & -12.28 & 9.36 & 0.19 & 0.19 & 6.35 & 0.98 \\
\hline Adjusted & -9.12 & 8.85 & 0.31 & 2.54 & 6.20 & 0.68 \\
\hline \multicolumn{7}{|c|}{$B M I<30 \mathrm{~kg} / \mathrm{m}^{2}(n=190)$} \\
\hline Crude & -7.60 & 5.32 & 0.16 & -8.52 & 5.03 & 0.09 \\
\hline Adjusted & -4.75 & 5.32 & 0.37 & -7.67 & 5.08 & 0.13 \\
\hline \multicolumn{7}{|c|}{$B M I \geq 30 \mathrm{~kg} / \mathrm{m}^{2}(n=76)$} \\
\hline Crude & -20.21 & 5.94 & 0.00 & 0.33 & 5.14 & 0.95 \\
\hline Adjusted & -20.41 & 6.51 & 0.00 & 1.36 & 5.38 & 0.80 \\
\hline
\end{tabular}

not alter these findings. Analyses restricted to weight gain cutoff at the 75th percentile revealed no additional insights as the patterns of association between H19 DMR methylation and IGF2 protein levels were similar to those of the analyses stratified by pre-pregnancy BMI (data not shown).

IGF2 DMR methylation and IGF2 protein concentrations

Table 3 also shows that hypomethylation at the $I G F 2$ DMR was significantly associated with higher IGF2 protein concentrations $(\beta=-9.87, p=0.01)$. Adjusting for maternal BMI pre-pregnancy strengthened this association $(\beta=$ $-11.36, p=0.01)$ and further adjusting for chorioamnionitis, birth weight and race/ethnicity did not alter this association. This association may be stronger in African Americans $(p=0.06)$ than Whites $(p=0.31)$. Analyses stratified by maternal BMI before pregnancy revealed an association between IGF2 DMR methylation and IGF2 protein levels, which was considerably stronger in infants born to obese women $(\beta=-20.41, p=0.003)$ corresponding to $\sim 110 \mathrm{ng} / \mu \mathrm{l}$ increase in IGF2 protein concentration for every $5 \%$ decrease in DNA methylation levels at the IGF2 DMR. The magnitude of this association was markedly weaker in infants born to non-obese women ( $\beta=-4.75, p=0.37)$. The cross-product term for obesity and IGF2 DMR methylation, however, was not statistically significant $(p=0.78)$. Because weight gain during pregnancy is strongly associated with pre-pregnancy BMI, repeating these analyses within each stratum of pre-pregnancy weight gain also did not reveal any insight to clarify the relative importance of these factors as the associations were much stronger in the 71 infants born to women with the largest weight gain ( $>75$ th percentile), when compared to those who gained $\leq 75$ th percentile. Intriguingly, including maternal weight gain in models with pre-pregnancy BMI and its cross-product term did not suggest weight gain was an important factor in the association between IGF2 DMR methylation and IGF2 protein concentration.

IGF2 protein concentrations and birth weight

The mean birth weight in this population $(n=300)$ was $3.14 \mathrm{~kg}$, with more than $95 \%$ of infants in the $2.2-5.2 \mathrm{~kg}$ range. We examined the association between IGF2 protein levels in UCB plasma and birth weight and found a linear increase in birth weight with increasing IGF2 protein levels $(\beta=0.28, \mathrm{SE}=0.07, p=0.0003)$ (Table 4). This association was free from the influence of factors known to be associated with birth weight including maternal race/ethnicity, pre-pregnancy BMI, education, gestational diabetes, cigarette smoking, and sex of infant. Table 4 also shows that while maternal cigarette smoking was independently but inversely associated with birth weight $(\beta=$ $-230.2, p$ value $=0.009)$, pre-pregnancy BMI $(\beta=197.4$, $p$ value $=0.01$ ) and Caucasian race/ethnicity (167.6, $p$ value $<0.0001)$ were positively associated with birth weight. Further inclusion of weight gain only widened confidence intervals, but did not alter risk estimates.

\section{Discussion}

We examined DNA methylation within two regulatory regions of imprinted IGF2 in relation to circulating IGF2 concentrations in UCB of newborns. We observed a 
Table 4 Regression model $\beta$-coefficients and standard errors of the association between umbilical cord blood IGF2 protein concentrations and birth weight $(n=278)$

\begin{tabular}{|c|c|c|c|}
\hline Characteristic/factor & $\beta$ & SE & $p$ value \\
\hline IGF2 protein concentrations $(\mathrm{ng} / \mu \mathrm{l})$ & 0.275 & 0.075 & 0.0003 \\
\hline Race/ethnicity (African American vs. Caucasian) & 167.6 & 39.57 & $<0.0001$ \\
\hline Maternal cigarette smoking & -230.17 & 88.57 & 0.0099 \\
\hline BMI before pregnancy (obese vs. non-obese) & 197.43 & 80.23 & 0.0145 \\
\hline Gestational diabetes & 137.35 & 125.28 & 0.2739 \\
\hline Sex of infant & -50.52 & 67.77 & 0.4566 \\
\hline
\end{tabular}

Sex, birth weight, or BMI could not be ascertained in 12 of 300 infants. Factors are mutually adjusted for each other. IGF2 protein levels are associated with higher birth weight $(p=0.0003)$; association is independent of race/ethnicity, maternal cigarette smoking, pre-pregnancy BMI, gestational diabetes, and sex

significant increase in IGF2 protein concentrations with decrease in methylation levels at the IGF2 DMR. This association was strongest among infants born to women with a pre-pregnancy $\mathrm{BMI}>30$, similar in African Americans and Caucasians. Effect sizes of a similar magnitude have been previously reported at the IGF2 DMR and other regions [26, 34, 35]. We also evaluated IGF2 protein concentrations in relation to birth weight, and observed elevated IGF2 protein concentrations were associated with a higher birth weight. We found no evidence for associations between CpG methylation at the H19 DMR and IGF2 protein concentration after adjusting for maternal obesity and race/ethnicity. We provide the first evidence for an association between DNA methylation at the IGF2 DMR and IGF2 protein concentrations. These findings are consistent with the interpretation that DNA methylation at the $I G F 2$ DMR is functionally relevant to the production of the potent mitogenic growth factor IGF2 and that soluble IGF2 independently contributes to variation in birth weight.

The exact mechanism by which loss of methyl groups at the IGF2 DMR increases IGF2 protein concentrations, exacerbated by maternal obesity, is still unknown, although changes in DNA methylation on the maternally derived allele has been shown to relax imprint controls, reactivating the otherwise silent maternally derived allele, which has been shown in previous studies to increase IGF2 mRNA and presumably protein products. Because imprinted genes are usually found in clusters [36] and their regulation may be networked [37], methylation alterations of this DMR have the potential for regulating (and dysregulating) multiple genes on different chromosomes. Therefore, methylation at this single DMR can provide information on epigenetic dysregulation elsewhere in the genome [18]. Using DNA obtained from leukocytes, methylation differences of a similar magnitude were reported among Gambians [17] and Dutch [26] adults who were exposed to nutritional challenges periconceptionally. Together, these findings support the idea that methylation marks acquired in the periconceptional period is similar across tissue types and is stable over many years. The stability of DNA methylation marks at the IGF2 DMR during a 3-year period was also recently reported among adult population controls [38]. The small magnitude of differences is also consistent with the contention that methylation marks acquired as a result of adjustments to perceived environment may not be as large as those found in cancer [39, 40]). Strong inverse associations between DNA methylation levels at the IGF2 DMR using DNA obtained from leukocytes, biallelic expression of this otherwise monoallelically expressed gene, and elevated colon cancer risk $[2,38,41]$ have been reported. In tissue specimens, hypermethylation at the H19 DMR is also frequent in cancers of the ovary [42], breast [43], esophagus [44], and prostate [45]. The IGF2 and HI9 DMRs have been previously proposed as biomarkers, biosensors, or archives of early exposures [2, 27, 28]. Therefore, our findings of a strong relationship between lower methylation at the IGF2 DMR and elevated IGF2 protein levels support the use of DNA methylation levels at this and perhaps other DMRs as biosensors or archives of early exposure. Differences observed by maternal obesity may be partly explained by genetic factors that also play a role in modulating IGF2 levels in the offspring.

Findings that protein levels of the mitogenic IGF2 are strongly associated with decreased methylation at the IGF2 DMR and with birth weight support the interpretation that variation in birth weight is modulated, at least in part, by $I G F 2$ plasticity. This may also be occurring at other DMRs, if hypothesized co-regulation with other imprinted regions [37] can be demonstrated. Previous studies have reported an association between circulating IGF2 protein concentrations and high birth weight [46] as well as risk of obesity [47] and several cancers [48, 49] in adulthood, suggesting early exposures may contribute to the variation in birth weight via epigenetic alterations.

Our inability to find a consistent association between H19 DMR methylation and IGF2 protein levels except among the 188 infants born to women with BMI $<30$ was 
unexpected. Current thinking for transcriptional control of the imprinted $I G F 2 / H 19$ domain correlates increased DNA methylation at the H19 DMR with failure to bind methylation sensitive CTCF, which can transcriptionally activate IGF2 on the normally silent maternal chromosome [50-52]. We cannot exclude the possibility that these were chance findings, since the statistical power to conduct multivariable analyses may have been inadequate. However, others have also failed to find a relationship between methylation at the H19 DMR and IGF2 transcription among children conceived in vitro and in vivo [52] suggesting that at the H19 DMR, other processes including genetic factors or other unmeasured environmental stimuli may contribute to increased IGF2 protein concentrations and body size, independent of DNA methylation alterations.

The main limitation of this study is the small sample size which, although more than adequate for examining crude relationships among DMR methylation fractions, protein IGF2 concentrations and birth weight, may have been inadequate for multivariable analyses, especially when testing interaction by race and maternal BMI. Despite the need for larger studies to clarify these relationships, the analyses raised the possibility that maternal obesity, which may be driven by other factors including genetic variation, also plays a role in modulating IGF2 concentrations. This suggests that a more detailed understanding of these epigenetic targets will require genetic analyses within the sequences under evaluation. Another limitation is that DNA obtained from unfractionated blood containing multiple cell types may have distinct epigenetic profiles depending on the cell population analyzed. However, we have previously described the analysis of fractionated cord blood for methylation at the same H19 and IGF2 DMRs, and the major cell types showed no significant differences in methylation levels [30]. Additionally, overall white blood cell counts vary and could confound the methylation fraction measures. However, inclusion of chorioamnionitis during parturition into statistical models did not alter our findings, suggesting variation in total cell counts may not contribute to differences in methylation at these DMRs. Furthermore, although we demonstrated an association between DMR methylation and protein levels downstream, we were limited by a lack of transcription data. However, a positive correlation between the levels of IGF2 mRNA and DNA methylation at these DMRs has been previously reported. These limitations notwithstanding, our findings suggest that IGF2 DMR plasticity is an important mechanism by which IGF2 protein levels are modulated.

In summary, among 300 newborns, we found a strong inverse association between DNA methylation at the $I G F 2$ regulatory sequence and elevated plasma protein concentrations of the encoded mitogenic growth factor. This relationship appeared to be stronger in infants born to women who were obese before pregnancy, but not weight gain. Higher IGF2 protein concentrations were also associated with higher birth weight. Herein, we provide evidence in support of the functional significance of aberrant methylation profiles at regulatory elements controlling the expression of imprinted $I G F 2$, supporting the use of $I G F 2$ DMR methylation as an archive or biosensor of early exposure. If co-regulation of imprinted domains can be demonstrated in humans, and transcripts are also associated with DNA methylation and protein levels for other imprinted genes in the same population, our findings would support the use of methylation marks in an imprint regulatory network to improve exposure assessment in studies of cancer and other chronic diseases.

Acknowledgments We thank the parents and children of the Newborn Epigenetics Study, research nurse Tammy Bishop, and research personnel: Stacy Murray, Carole Grenier, Darby Kroyer, Natasha Duggan, Suba Narasimhan, and James Nathan Yarnall for enrolling and tracing participants, and processing the specimens; and the obstetrics faculty and staff at Duke University and Durham regional Hospitals, Durham, NC. This work was supported in part by R21ES014947, R01ES016772, R01DK085173, K01CA104517 the American Cancer Society (grant number ACS-IRG-83-006) and the Fred and Alice Stanback Foundation. Dr. Susan Murphy was also supported by the Duke Comprehensive Cancer Center and Duke Environmental Health Sciences Research Center.

Conflict of interest The authors declare that they have no conflict of interest.

Open Access This article is distributed under the terms of the Creative Commons Attribution License which permits any use, distribution, and reproduction in any medium, provided the original author(s) and the source are credited.

\section{References}

1. Bateson P, Barker D, Clutton-Brock T et al (2004) Developmental plasticity and human health. Nature 430:419-421

2. Cui H, Cruz-Correa M, Giardiello FM et al (2003) Loss of IGF2 imprinting: a potential marker of colorectal cancer risk. Science 299:1753-1755 New York, NY

3. Nowakowska-Zajdel E, Mazurek U, Ziolko E et al (2011) Analysis of expression profile of gene encoding proteins of signal cascades activated by insulin-like growth factors in colorectal cancer. Int J Immunopathol Pharmacol 24:781-787

4. Murphy SK, Huang Z, Wen Y et al (2006) Frequent IGF2/H19 domain epigenetic alterations and elevated IGF2 expression in epithelial ovarian cancer. Mol Cancer Res 4:283-292

5. Jarrard DF, Bussemakers MJ, Bova GS, Isaacs WB (1995) Regional loss of imprinting of the insulin-like growth factor II gene occurs in human prostate tissues. Clin Cancer Res 1:1471-1478

6. Bhusari S, Yang B, Kueck J, Huang W, Jarrard DF (2011) Insulin-like growth factor-2 (IGF2) loss of imprinting marks a field defect within human prostates containing cancer. Prostate 71:1621-1630

7. Zuo QS, Yan R, Feng DX et al (2011) Loss of imprinting and abnormal expression of the insulin-like growth factor 2 gene in gastric cancer. Mol Carcinog 50:390-396 
8. Xu W, Fan H, He X, Zhang J, Xie W (2006) LOI of IGF2 is associated with esophageal cancer and linked to methylation status of IGF2 DMR. J Exp Clin Cancer Res 25:543-547

9. Wu AH, McKean-Cowdin R, Tseng CC (2011) Birth weight and other prenatal factors and risk of breast cancer in Asian-Americans. Breast Cancer Res Treat 130:917-925

10. Forman MR, Cantwell MM, Ronckers C, Zhang Y (2005) Through the looking glass at early-life exposures and breast cancer risk. Cancer Invest 23:609-624

11. Bagchi S (2005) Intrauterine growth linked to aggressive prostate cancer. Lancet Oncol 6:70

12. Eriksson M, Wedel H, Wallander MA et al (2007) The impact of birth weight on prostate cancer incidence and mortality in a population-based study of men born in 1913 and followed up from 50 to 85 years of age. Prostate 67:1247-1254

13. Nilsen TI, Romundstad PR, Troisi R, Vatten LJ (2005) Birth size and subsequent risk for prostate cancer: a prospective populationbased study in Norway. Int J Cancer 113:1002-1004

14. Akre O, Forssell L, Kaijser M et al (2006) Perinatal risk factors for cancer of the esophagus and gastric cardia: a nested casecontrol study. Cancer Epidemiol Biomarkers Prev 15:867-871

15. Kaijser M, Akre O, Cnattingius S, Ekbom A (2005) Preterm birth, low birth weight, and risk for esophageal adenocarcinoma. Gastroenterology 128:607-609

16. Strathdee G, Sim A, Brown R (2004) Control of gene expression by $\mathrm{CpG}$ island methylation in normal cells. Biochem Soc Trans 32:913-915

17. Waterland RA, Kellermayer R, Laritsky E et al (2010) Season of conception in rural gambia affects DNA methylation at putative human metastable epialleles. PLoS Genet 6:e1001252

18. Woodfine K, Huddleston JE, Murrell A (2011) Quantitative analysis of DNA methylation at all human imprinted regions reveals preservation of epigenetic stability in adult somatic tissue. Epigenetics Chromatin 4:1

19. Murphy SK, Jirtle RL (2003) Imprinting evolution and the price of silence. Bioassays 25:577-588

20. Murrell A, Ito Y, Verde G et al (2008) Distinct methylation changes at the IGF2-H19 locus in congenital growth disorders and cancer LOI of IGF2 is associated with esophageal cancer and linked to methylation status of IGF2 DMR. PLoS ONE 3:e1849

21. Cui H, Niemitz EL, Ravenel JD et al (2001) Loss of imprinting of insulin-like growth factor-II in Wilms' tumor commonly involves altered methylation but not mutations of CTCF or its binding site. Cancer Res 61:4947-4950

22. Cui H, Onyango P, Brandenburg S, Wu Y, Hsieh CL, Feinberg AP (2002) Loss of imprinting in colorectal cancer linked to hypomethylation of H19 and IGF2. Cancer Res 62:6442-6446

23. Nakagawa H, Chadwick RB, Peltomaki P, Plass C, Nakamura Y, de La Chapelle A (2001) Loss of imprinting of the insulin-like growth factor II gene occurs by biallelic methylation in a core region of H19-associated CTCF-binding sites in colorectal cancer. Proc Natl Acad Sci U S A 98:591-596

24. Sullivan MJ, Taniguchi T, Jhee A, Kerr N, Reeve AE (1999) Relaxation of IGF2 imprinting in Wilms tumours associated with specific changes in IGF2 methylation. Oncogene 18:7527-7534

25. Takai D, Gonzales FA, Tsai YC, Thayer MJ, Jones PA (2001) Large scale mapping of methylcytosines in CTCF-binding sites in the human $\mathrm{H} 19$ promoter and aberrant hypomethylation in human bladder cancer. Hum Mol Genet 10:2619-2626

26. Heijmans BT, Tobi EW, Stein AD et al (2008) Persistent epigenetic differences associated with prenatal exposure to famine in humans. Proc Natl Acad Sci U S A 105:17046-17049

27. Hoyo C, Murphy SK, Jirtle RL (2009) Imprint regulatory elements as epigenetic biosensors of exposure in epidemiological studies. J Epidemiol Community Health 63:683-684
28. Heijmans BT, Tobi EW, Lumey LH, Slagboom PE (2009) The epigenome: archive of the prenatal environment. Epigenetics 4:526-531

29. Hoyo C, Murtha AP, Schildkraut JM et al (2011) Folic acid supplementation before and during pregnancy in the newborn epigenetics study (NEST). BMC Public Health 11:46

30. Hoyo C, Murtha AP, Schildkraut JM et al (2011) Methylation variation at IGF2 differentially methylated regions and maternal folic acid use before and during pregnancy. Epigenetics 6: 928-936

31. Huang Z, Wen Y, Shandilya R, Marks JR, Berchuck A, Murphy SK (2006) High throughput detection of M6P/IGF2R intronic hypermethylation and LOH in ovarian cancer. Nucleic Acids Res 34:555-563

32. Umbers AJ, Boeuf P, Clapham C et al (2011) Placental malariaassociated inflammation disturbs the insulin-like growth factor axis of fetal growth regulation. J Infect Dis 203:561-569

33. Schlinzig T, Johansson S, Gunnar A, Ekstrom TJ, Norman M (2009) Epigenetic modulation at birth-altered DNA-methylation in white blood cells after caesarean section. Acta Paediatr 98: 1096-1099

34. Steegers-Theunissen RP, Obermann-Borst SA, Kremer D et al (2009) Periconceptional maternal folic acid use of 400 microg per day Is related to increased methylation of the IGF2 gene in the very young child. PLoS ONE 4:e7845

35. Talens RP, Boomsma DI, Tobi EW et al (2010) Variation, patterns, and temporal stability of DNA methylation: considerations for epigenetic epidemiology. FASEB J 24:3135-3144

36. Edwards CA, Rens W, Clarke O et al (2007) The evolution of imprinting: chromosomal mapping of orthologues of mammalian imprinted domains in monotreme and marsupial mammals. BMC Evol Biol 7:157

37. Varrault A, Gueydan C, Delalbre A et al (2006) Zac1 regulates an imprinted gene network critically involved in the control of embryonic growth. Dev Cell 11:711-722

38. Cruz-Correa M, Zhao R, Oviedo M et al. (2009) Temporal stability and age-related prevalence of loss of imprinting of the insulin-like growth factor-2 gene. Epigenetics 4:114-118

39. Jirtle RL, Skinner MK (2007) Environmental epigenomics and disease susceptibility. Nat Rev 8:253-262

40. Feinberg AP (2007) An epigenetic approach to cancer etiology. Cancer J 13:70-74 (SudburyMass)

41. Cruz-Correa M, Cui H, Giardiello FM et al (2004) Loss of imprinting of insulin growth factor II gene: a potential heritable biomarker for colon neoplasia predisposition. Gastroenterology 126:964-970

42. Dammann RH, Kirsch S, Schagdarsurengin U et al (2010) Frequent aberrant methylation of the imprinted IGF2/H19 locus and LINE1 hypomethylation in ovarian carcinoma. Int $\mathbf{J}$ Oncol 36: 171-179

43. Yang R, Wang Y, Zhu X, Gao X (2009) Study on genomic imprinting of IGF2 in breast cancer. Wei sheng yan jiu. J Hyg Res 38:423-425

44. Zhao R, DeCoteau JF, Geyer CR, Gao M, Cui H, Casson AG (2009) Loss of imprinting of the insulin-like growth factor II (IGF2) gene in esophageal normal and adenocarcinoma tissues. Carcinogenesis 30:2117-2122

45. Paradowska A, Fenic I, Konrad L et al (2009) Aberrant epigenetic modifications in the CTCF binding domain of the IGF2/H19 gene in prostate cancer compared with benign prostate hyperplasia. Int J Oncol 35:87-96

46. Johnsen SP, Sorensen HT, Thomsen JL et al (2004) Markers of fetal growth and serum levels of insulin-like growth factor (IGF) I, -II and IGF binding protein 3 in adults. Eur J Epidemiol 19: $41-47$ 
47. Fowke JH, Matthews CE, Yu H et al (2010) Racial differences in the association between body mass index and serum IGF1, IGF2, and IGFBP3. Endocr Relat Cancer 17:51-60

48. Gronbaek H, Flyvbjerg A, Mellemkjaer L et al (2004) Serum insulin-like growth factors, insulin-like growth factor binding proteins, and breast cancer risk in postmenopausal women. Cancer Epidemiol Biomarkers Prev 13:1759-1764

49. Shetty PJ, Movva S, Pasupuleti N et al (2011) Regulation of IGF2 transcript and protein expression by altered methylation in breast cancer. J Cancer Res Clin Oncol 137:339-345
50. Murphy SK, Wylie AA, Coveler KJ et al (2003) Epigenetic detection of human chromosome 14 uniparental disomy. Hum Mutat 22:92-97

51. Murrell A, Ito Y, Verde G et al (2008) Distinct methylation changes at the IGF2-H19 locus in congenital growth disorders and cancer. PLoS ONE 3:e1849

52. Turan N, Katari S, Gerson LF et al (2010) Inter- and intra-individual variation in allele-specific DNA methylation and gene expression in children conceived using assisted reproductive technology. PLoS Genet 6:e1001033 